\title{
Emittance Growth from Electron Beam Modulation
}

M. Blaskiewicz

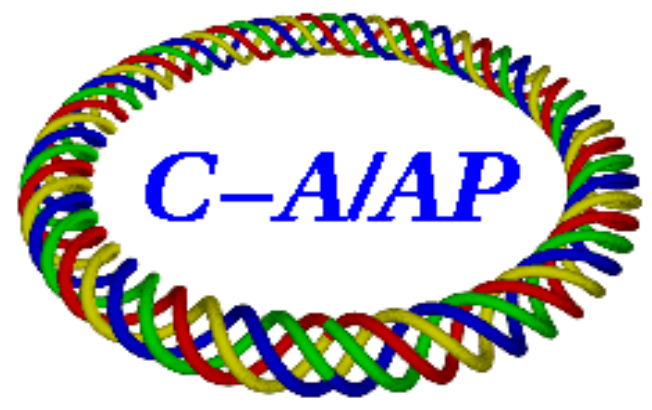

Collider-Accelerator Department Brookhaven National Laboratory

Upton, NY 11973

Notice: This document has been authorized by employees of Brookhaven Science Associates, LLC under Contract No. DE-AC02-98CH10886 with the U.S. Department of Energy. The United States Government retains a nonexclusive, paid-up, irrevocable, world-wide license to publish or reproduce the published form of this document, or allow others to do so, for United States Government purposes. 


\title{
Emittance Growth from Electron Beam Modulation
}

\author{
M. Blaskiewicz* \\ $B N L$, Upton NY 11973, USA
}

\begin{abstract}
In linac ring colliders like MeRHIC and eRHIC a modulation of the electron bunch can lead to a modulation of the beam beam tune shift and steering errors [1-3]. These modulations can lead to emittance growth. This note presents simple formulas to estimate these effects which generalize some previous results.
\end{abstract}

\section{THEORY OF QUADRUPOLE GROWTH}

Consider one dimensional motion in the $x$ direction with bare tune $Q$. We use the evolution variable $\theta$ which updates by $2 \pi$ each turn. The interaction point is at $\theta=0$, the modulating beam beam force is denoted by $\epsilon(\theta)$ and the hamiltonian is given by

$$
H(x, p, \theta)=\frac{p^{2}+Q^{2} x^{2}}{2}+\delta_{p}(\theta) \frac{Q x^{2}}{2} \epsilon(\theta),
$$

where the periodic delta function is

$$
\delta_{p}(\theta)=\sum_{k=-\infty}^{\infty} \delta(\theta-2 \pi k)
$$

The equations of motion are $d x / d \theta=\partial H / \partial p$ and $d p / d \theta=-\partial H / \partial x$. Do a canonical transformation to action angle variables with $x=\sqrt{2 J / Q} \sin \psi$ and $p=\sqrt{2 J Q} \cos \psi$ yielding

$$
H(\psi, J, \theta)=Q J+\delta_{p}(\theta) J \sin ^{2} \psi \epsilon(\theta) .
$$

Averaging over $\psi$ and $\epsilon$ with $\left\langle\delta_{p}(\theta)\right\rangle=1 / 2 \pi$ one finds $\langle H(J)\rangle=Q J+J\langle\epsilon\rangle / 4 \pi$ so the average beam beam tune shift is $\langle\delta Q\rangle=\langle\epsilon>/ 4 \pi$. We redefine the tune to be $Q \rightarrow Q+<\delta Q>$ and introduce the fluctuating part of the noise. $\delta \epsilon=\epsilon-\langle\epsilon\rangle$. The hamiltonian becomes

$$
H(\psi, J, \theta)=Q J+\delta_{p}(\theta) J \sin ^{2} \psi \delta \epsilon(\theta) .
$$

The emittance growth is due to the modulation in $\delta \epsilon$ and the equations of motion are

$$
\frac{d J}{d \theta}=-\frac{\partial H}{\partial \psi}=-\delta_{p}(\theta) J \sin (2 \psi) \delta \epsilon(\theta)
$$

and

$$
\frac{d \psi}{d \theta}=\frac{\partial H}{\partial J}=Q+\delta_{p}(\theta) \sin ^{2} \psi \delta \epsilon(\theta)
$$

The expansion parameter is $\delta \epsilon$. To zeroeth order $\psi_{0}(\theta)=Q \theta+\hat{\psi}$. To first order in $\delta \epsilon$

$$
\begin{aligned}
\psi_{1}(\theta) & =Q \theta+\hat{\psi}+\int_{0}^{\theta} \delta_{p}(s) \sin ^{2}(Q s+\hat{\psi}) \delta \epsilon(s) d s \\
& \approx Q \theta+\hat{\psi}-\frac{1}{2} \int_{0}^{\theta} \delta_{p}(s) \cos (2 Q s+2 \hat{\psi}) \delta \epsilon(s) d s .
\end{aligned}
$$

*Electronic address: blaskiewicz@bnl.gov 
The evolution of the action is given by

$$
\begin{aligned}
\frac{d \ln J}{d \theta} & =-\delta_{p}(\theta) \sin (2 \psi) \delta \epsilon(\theta) \\
& \approx-\delta_{p}(\theta) \sin \left(2 \psi_{1}(\theta)\right) \delta \epsilon(\theta) \\
& \approx-\delta_{p}(\theta) \sin (2 Q \theta+2 \hat{\psi}) \delta \epsilon(\theta) \\
& +\delta_{p}(\theta) \cos (2 Q \theta+2 \hat{\psi}) \delta \epsilon(\theta) \int_{0}^{\theta} \delta_{p}(s) \cos (2 Q s+2 \hat{\psi}) \delta \epsilon(s) d s \\
& \approx-\delta_{p}(\theta) \sin (2 Q \theta+2 \hat{\psi}) \delta \epsilon(\theta) \\
& +\frac{1}{2} \frac{d}{d \theta}\left[\int_{0}^{\theta} \delta_{p}(s) \cos (2 Q s+2 \hat{\psi}) \delta \epsilon(s) d s\right]
\end{aligned}
$$

Integrating equation (9) one finds $J(\theta)=J(0) \exp (u(\theta))$ where

$$
u(\theta)=-\int_{0}^{\theta} \delta_{p}(s) \sin (2 Q s+2 \hat{\psi}) \delta \epsilon(s)+\frac{1}{2}\left[\int_{0}^{\theta} \delta_{p}(s) \cos (2 Q s+2 \hat{\psi}) \delta \epsilon(s) d s\right]^{2} .
$$

As long as $u$ is small compared to one, which is necessary for eq (8), the first term on the right hand side dominates. Hence $u$ is effectively the sum of a large number of uncorrelated random variables and therefore nearly a gaussian random variable. Notice that

$$
<u(\theta)\rangle=\left\langle\frac{1}{2}\left[\int_{0}^{\theta} \delta_{p}(s) \cos (2 Q s+2 \hat{\psi}) \delta \epsilon(s) d s\right]^{2}\right\rangle \approx \sigma_{u}^{2} / 2
$$

Now since $\left\langle\exp (u)>=\exp \left(<u>+\sigma_{u}^{2} / 2\right)\right.$ for a gaussian, we have $<J(\theta)>=J(0) \exp (2<u(\theta)>)$ and the problem is to evaluate $\langle u(\theta)\rangle$. Start by using the periodic delta function to turn the integral to a sum and define $\delta \epsilon(2 \pi k)=\delta \epsilon_{k}$ so that

$$
\begin{aligned}
<2 u_{n}> & =\sum_{k=0}^{n} \sum_{m=0}^{n} \cos (4 \pi Q k+2 \hat{\psi}) \cos (4 \pi Q m+2 \hat{\psi})<\delta \epsilon_{k} \delta \epsilon_{m}> \\
& \approx \sum_{k=0}^{n} \sum_{m=0}^{n} \frac{1}{2} \cos (4 \pi Q[k-m])<\delta \epsilon_{k} \delta \epsilon_{m}> \\
& \approx n \sum_{m=-\infty}^{\infty} \frac{1}{2} \cos (4 \pi Q m)<\delta \epsilon_{k} \delta \epsilon_{m+k}>
\end{aligned}
$$

where $\delta \epsilon_{k}$ is assumed to be stationary noise. As a simple model take $\left\langle\delta \epsilon_{k} \delta \epsilon_{m+k}>=\sigma_{\epsilon}^{2} \exp (-\alpha|m|)\right.$. In this case the sum can be done resulting in

$$
\ln \left\langle\frac{J(\theta)}{J(0)}\right\rangle=<2 u_{n}>=n \frac{\sigma_{\epsilon}^{2}}{2} \frac{1-e^{-2 \alpha}}{1+e^{-2 \alpha}-2 \cos (4 \pi Q) e^{-\alpha}}
$$

\section{COMPARISON WITH SIMULATIONS}

For simulation purposes I take a one turn matrix followed by a thin lens beam beam kick,

$$
\begin{aligned}
& x_{n}(k+1)=\cos \psi_{n} x_{n}(k)+\sin \psi_{n} p_{n}(k) \\
& p_{n}(k+1)=\cos \psi_{n} p_{n}(k)-\sin \psi_{n} x_{n}(k)+\epsilon_{k} x_{n}(k+1)
\end{aligned}
$$




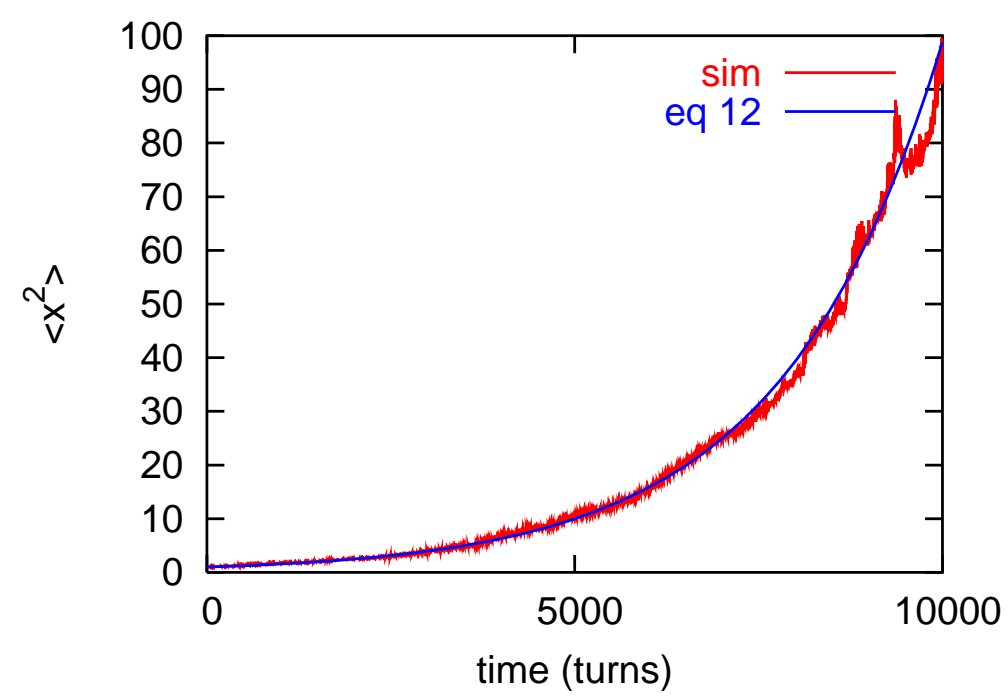

FIG. 1: Comparison of simulation and equation 12. The correlation suppression factor is 0.06 .

where $k$ is the time like variable and each of the $n$ particles has a slightly different phase advance to create filamentation. The beam beam kick $\epsilon_{k}$ is normalized as above. Comparison of a simulation and equation (13) is shown in Fig 1. The correlation time of the noise was $\alpha=0.1$ and $Q=0.2$ resulting in a growth rate only 0.06 of what it would be for white noise of the same amplitude. As one can see from the figure the agreement is very good over many e-folding times. Of course in a real beam the electron kick is not purely linear so the growth would be reduced. In any case the formula seems reliable for an e-folding time.

\section{GROWTH DUE TO STEERING ERRORS}

Along with variations in electron beam intensity and emittance there can also be steering errors. We start by writing the one turn map in the linear approximation

$$
\begin{aligned}
x(k+1) & =\cos \psi x(k)+\sin \psi x^{\prime}(k) \beta^{*} \\
x^{\prime}(k+1) & =\cos \psi x^{\prime}(k)-\sin \psi x(k) / \beta^{*}-\epsilon[x(k+1)-y(k+1)] / \beta^{*}
\end{aligned}
$$

where $y(k)$ is the electron beam offset, $\beta^{*}$ is the beta function at the crossing point, and the beam beam tune shift is $\epsilon / 4 \pi$ as before. We neglect the beam beam tune shift and define $z(k)=x(k)+i \beta^{*} x^{\prime}(k)$ then

$$
z(k+1)=e^{-i \psi} z(k)+i \epsilon y(k+1) .
$$

Set $U(k)=z(k) \exp (i k \psi)$ yielding

$$
U(k+1)=U(k)+i \epsilon y(k+1) \exp (i(k+1) \psi),
$$

so that

$$
U(k)=U_{0}+i \epsilon \sum_{m=0}^{k} y(m) \exp (i m \psi) .
$$

Taking averages

$$
\begin{aligned}
<|U(k)|^{2}> & =\left|U_{0}\right|^{2}+\epsilon^{2} \sum_{m} \sum_{\ell}<y(m) y(\ell)>\exp (i(m-\ell) \psi) \\
& \approx\left|U_{0}\right|^{2}+k \epsilon^{2} \sum_{m=-\infty}^{\infty}<y(m+\ell) y(\ell)>\cos (m \psi)
\end{aligned}
$$


Taking $<y(m+\ell) y(\ell)>=\sigma_{y}^{2} \exp (-\alpha|m|)$ one finds

$$
2 \epsilon_{r m s} \beta^{*}=<|U(k)|^{2}>=\left|U_{0}\right|^{2}+k\left(\epsilon \sigma_{y}\right)^{2} \frac{1-e^{-2 \alpha}}{1+e^{-2 \alpha}-2 \cos (2 \pi Q) e^{-\alpha}},
$$

where $\epsilon_{r m s}=<x^{2}>/ \beta^{*}$ is the rms geometric emittance of the ion beam.

\section{Acknowledgements}

Thanks to Vadim Ptitsyn for useful discussions and encouragement.

[1] C. Montag, RHIC/AP/143, 2004 also C. Montag NIM A, Volume 581, p581, 2007

[2] See Eduard Pozdeyev's MeRHIC discussion September 7, 2006.

[3] Y. Hao, V. Litvinenko, C. Montag, E. Pozdeyev, V. Ptitsyn, PAC07, p1865 (2007). 